\title{
Controversias en el tratamiento de la hernia de Amyand
}

\author{
Controversies in the treatment of Amyand's hernia
}

\author{
María José Servide-Staffolani1,6, Arkaitz Perfecto-Valero1, Jorge Cervera-Aldama², \\ María Fernanda Anduaga-Peña ${ }^{3,6}$, José María García-González ${ }^{4}$, Alberto Colina-Alonso ${ }^{5}$, \\ Patrizio Petrone $e^{6,7}$
}

${ }^{1}$ Servicio de Cirugía General, Hospital Universitario de Cruces, Barakaldo, Vizcaya, España

${ }^{2}$ Unidad de Coloproctología, Servicio de Cirugía General, Hospital Universitario de Cruces, Barakaldo, Vizcaya, España

${ }^{3}$ Servicio de Cirugía General, Hospital Universitario de Salamanca, Salamanca, España

${ }^{4}$ Jefe, Unidad de Coloproctología, Servicio de Cirugía General, Hospital Universitario de Cruces, Barakaldo, Vizcaya, España

${ }_{6}^{5}$ Jefe, Servicio de Cirugía General, Hospital Universitario de Cruces, Barakaldo, Vizcaya, España

${ }^{6}$ Department of Surgery, NYU-Winthrop Hospital, Mineola, New York, USA

${ }^{7}$ Clinical Associate Professor of Surgery, New York Medical College, New York, USA

\section{Resumen}

La hernia de Amyand tiene baja incidencia (o,I a I \%) pero, en caso de complicación, puede asociarse con una alta tasa de mortalidad. A pesar de haber sido descrita hace más de 200 años por Claudius Amyand, siguen existiendo ciertas controversias sobre su correcto tratamiento en determinadas situaciones.

La práctica o la evitación de la apendicectomía en ausencia de apendicitis y la técnica para reparar el defecto herniario según el grado de contaminación de la zona, son temas tratados en el presente artículo. Ante la falta de consenso global en la literatura científica, la individualización de cada caso y el buen juicio del cirujano deben primar a la hora de decidir el tratamiento más adecuado.

Se presentan dos casos clínicos que, a pesar de tratarse de la misma enfermedad, tuvieron formas de presentación, manejo y evolución dispares. Asimismo, se adjunta la iconografía de un excepcional caso de hernia de Amyand, izquierda, junto con el hallazgo de una hernia de Littré no complicada en el mismo saco herniario.

Palabras clave: hernia inguinal; hernia de Amyand; hernia de Littre; apendicectomía; herniorrafia; prótesis e implantes.

\begin{abstract}
Amyand's hernia has a low incidence (0.1\%-I.0\%) but it may be associated with high mortality rates. Despite having been described more than 200 years ago by Claudius Amyand, there are still controversies regarding treatment in some particular situations. Performing or not appendectomies in the absence of appendicitis, or how to perform the hernia repair depending on the degree of contamination, are topics discussed in this article. There is not a global consensus in the literature yet. The individualization of each case and the surgeon's proper judgment should be the priority for selecting the most appropriate treatment. Two clinical cases of Amyand's hernia are reported with very different clinical presentations, management and evolution. Also, an image of a left sided Amyand's hernia together with a Littre's hernia in the same hernia sac is shown.

Fecha de recibido: 22/06/2017 Fecha de aceptado: 04/07/2017

Correspondencia: Patrizio Petrone, MD / Department of Surgery, NYU Winthrop Hospital, 222 Station Plaza North, Suite 603, Mineola, Long Island, New York 11501, USA. / Correo electrónico: patrizio.petrone@gmail.com y ppetrone@nyuwinthrop.org

Citar como: Servide-Staffolani MJ, Perfecto-Valero A, Cervera-Aldama J, Anduaga-Peña MF, García-González JM, Colina-Alonso A, Petrone P. Controversias en el tratamiento de la hernia de Amyand y presentación de dos casos. Rev Colomb Cir. 2018;33:107-10.
\end{abstract}


Keywords: Hernia, Inguinal; Amyand's hernia; Littre's hernia; Appendectomy; Herniorrhaphy; Prostheses and Implants.

\section{Introducción}

Se conoce como hernia de Amyand a la presencia del apéndice vermiforme en el interior del saco herniario inguinal, asociada o no a apendicitis. Claudius Amyand practicó, en I736, la primera apendicectomía por apendicitis perforada en una hernia inguinal en un niño de II años. No obstante, fue Croissant de Garengeot quien, en I73I, describió por primera vez la presencia del apéndice en el saco herniario ${ }^{1,2}$.

La hernia de Amyand presenta una incidencia del I \%, y cerca del o, I \% de los casos se asocian con apendicitis. Es más frecuente en niños varones y en hernias del lado derecho. La hernia de Amyand, izquierda, se relaciona con la presencia de un ciego móvil, situs inversus o mala rotación intestinal ${ }^{\mathrm{I}-3}$. Cuando se presenta con un cuadro clínico de peritonitis, se asocia con una alta tasa de mortalidad, que varía entre 4 y $30 \% 3$. El tratamiento se basa en la apendicectomía y en la reparación del defecto herniario. No obstante, siguen existiendo ciertas controversias sobre su manejo en determinadas situaciones ${ }^{4-6}$. A continuación se presentan dos casos clínicos.

\section{Caso 1}

Se trata de un hombre de 80 años de edad, con antecedentes de carcinoma de próstata en tratamiento médico y cardiopatía moderada, que consultó al Servicio de Urgencias por presentar dolor y tumoración inguinal de ocho días de evolución.

En el examen físico se observó una hernia inguinal derecha incarcerada y en la ecografía se describió el extremo apendicular inflamado en su interior. Con diagnóstico de hernia de Amyand, se decidió practicar una intervención quirúrgica urgente.

Durante la cirugía, se encontró una hernia crural incarcerada que contenía el apéndice cecal con cambios isquémicos y sin signos de apendicitis aguda. Se decidió practicar apendicecto- mía y hernioplastia crural según la técnica de Liechtenstein con malla de polipropileno. El paciente fue dado de alta, sin que hubiera presentado complicaciones asociadas.

\section{Caso 2}

Se trata de un hombre de 89 años de edad, con antecedentes de hipertensión arterial sistémica, cardiopatía por arritmia y broncopatía crónica grave. Acudió al Servicio de Urgencias por malestar general, y se encontraba sudoroso, frío e hipotenso a su llegada. Refirió molestia abdominal generalizada y vómitos.

En el examen físico, presentaba una hernia inguinal izquierda gigante no reducible, sin signos de peritonitis. En los exámenes de laboratorio se encontró aumento de los reactantes de fase aguda, acidosis metabólica y elevación del lactato. Ante la sospecha de sepsis de origen abdominal, se ordenó una tomografía computadorizada (TC), la cual mostró una gran hernia inguino-escrotal izquierda con el apéndice inflamado en su interior.

Los hallazgos intraoperatorios fueron de apendicitis aguda purulenta en una hernia inguino-escrotal gigante, que contenía el ciego y, aproximadamente, $100 \mathrm{~cm}$ de íleon terminal, con la presencia de un divertículo de Meckel no complicado (figura). Se practicó apendicectomía y cierre del orificio inguinal profundo, sin malla.

El paciente presentó una evolución posoperatoria tórpida, con falla multiorgánica, hipotensión y anuria, y falleció a las 48 horas de la cirugía.

\section{Discusión}

El tratamiento clásico de la hernia de Amyand se basa en la apendicectomía y en la reparación de la hernia. No obstante, sigue faltando consenso sobre el tratamiento óptimo en determinadas situaciones ${ }^{4-6}$. 
Losanoff y Bassoon clasificaron la hernia de Amyand en cuatro tipos y asociaron un determinado tratamiento para uno de ellos (tabla) ${ }^{6,7}$. Según su clasificación, en ausencia de signos de inflamación no sería necesaria la apendicectomía profiláctica y solo la recomiendan en pacientes jóvenes o niños. Por el contrario, en un trabajo en el cual se analizaron 46 casos de hernia de Amyand en pacientes pediátricos, los autores no la recomiendan cuando el apéndice se encuentra sano ${ }^{8}$.

Los detractores de la apendicectomía profiláctica apoyan la idea de que la reparación de la hernia es prioritaria en ausencia de apendicitis y que dicha intervención se asocia con ciertos factores que podrían poner en riesgo el éxito de la hernioplastia, como la infección de la malla, entre otros $1,3,8,9$.

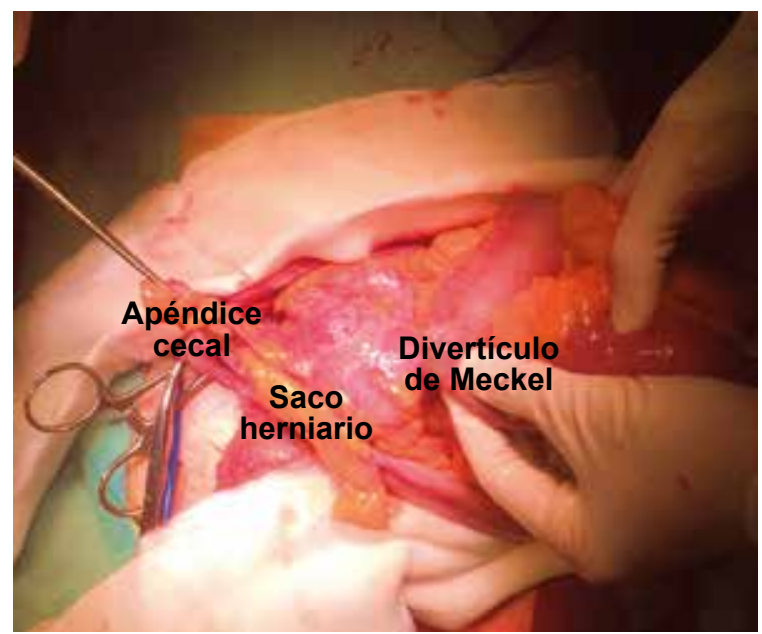

Figura. Hernia de Amyand a la izquierda y hernia de Littré
Por otra parte, los autores que apoyan la apendicectomía describen una menor tasa de morbimortalidad y se basan en la teoría de que la manipulación del apéndice durante la reparación de la hernia puede dar lugar a la evolución de una apendicitis en el futuro, y en que la colocación de la malla resulta segura en ausencia de apendicitis ${ }^{5,6}$. También la recomiendan en casos de hernia de Amyand, izquierda, en la cual una apendicitis podría dar lugar a un diagnóstico y tratamiento tardíos debido a su localización ${ }^{1,5}$.

En las hernias de Amyand que conllevan cierto grado de inflamación, estarían indicadas la apendicectomía y posterior reparación del defecto herniario evitando el uso de material protésico, debido al riesgo de fistula del muñón apendicular y de infección de la malla ${ }^{2,3,6,7,10}$. No obstante, el uso de la malla sigue siendo motivo de debate. Algunos autores la recomiendan solo en ausencia de perforación o necrosis apendicular, siempre con antibioticoterapia durante tres a cinco días ${ }^{1}$. En las hernias de tipo 2, podrían estar indicadas las mallas biológicas, dado el menor riesgo de recurrencia que con la reparación primaria sin malla, y el menor riesgo de infección en comparación con las mallas sintéticas ${ }^{3,6,7,10}$. Existe bajo nivel de evidencia y de resultados a largo plazo sobre el uso de mallas biológicas en estos casos, por lo que aún no puede considerarse como recomendación para el tratamiento de la hernia de Amyand de tipo $2^{3}$.

\section{Conclusión}

A pesar de las controversias sobre el manejo de esta patología, la individualización de cada caso

Tabla. Clasificación de la hernia de Amyand y su respectivo manejo ${ }^{8}$

\begin{tabular}{|c|c|c|c|c|}
\hline Tipo de hernia & 1 & 2 & 3 & 4 \\
\hline Características & Apéndice normal & $\begin{array}{l}\text { Apendicitis aguda } \\
\text { localizada en el saco } \\
\text { herniario }\end{array}$ & $\begin{array}{l}\text { Apendicitis aguda con } \\
\text { peritonitis o sepsis } \\
\text { abdominal }\end{array}$ & $\begin{array}{l}\text { Apendicitis aguda aso- } \\
\text { ciada a otra enferme- } \\
\text { dad intraabdominal }\end{array}$ \\
\hline $\begin{array}{l}\text { Tratamiento } \\
\text { quirúrgico }\end{array}$ & $\begin{array}{l}\text { Reducción de la hernia, } \\
\text { (apendicectomía en } \\
\text { pacientes jóvenes) y her- } \\
\text { nioplastia con malla }\end{array}$ & $\begin{array}{l}\text { Apendicectomía a } \\
\text { través de la hernia y } \\
\text { reparación sin malla } \\
\text { de la hernia }\end{array}$ & $\begin{array}{l}\text { Laparotomía, apendicec- } \\
\text { tomía y reparación sin } \\
\text { malla de la hernia }\end{array}$ & $\begin{array}{l}\text { Apendicectomía y trata- } \\
\text { miento específico de la } \\
\text { enfermedad asociada } \\
\text { en cada caso }\end{array}$ \\
\hline
\end{tabular}


y el buen juicio del cirujano deben primar a la hora de decidir el tratamiento más adecuado ${ }^{2}$.

La publicación de distintos casos clínicos de hernia de Amyand, con detalles sobre las formas de presentación clínica, las técnicas diagnósticas, las opciones terapéuticas y los resultados, es una herramienta que aporta información y puede ser de gran ayuda en la decisión de su manejo por parte de la comunidad quirúrgica internacional.

Asimismo, son de fundamental importancia los ensayos clínicos que aporten mayor información a largo plazo, para poder recomendar la utilización de mallas biológicas en las hernias de Amyand de tipo 2.

\section{Referencias}

I. Castro D, Borráez AM, Farías AP, Muñoz LM. Variante de una hernia de Amyand. Rev Colomb Cir. 2016;31:276-82.

2. Villarreal R, Jaspe CL, Cabrera LF, Vinck E. Hernia de Amyand encarcelada, revisión de la literatura y reporte de un caso en una institución de tercer nivel de Bogotá. Rev Colomb Cir. 2016;3I:283-8.

3. Morales-Cárdenas A, Ploneda-Valencia CF, Sainz-Escárrega VH, Hernández-Campos AC, Navarro-Muñiz
E, López-Lizarraga CR, et al. Amyand hernia: Case report and review of the literature. Ann Med Surg. 2015;4:I13-5. doi: I0.IOI6/j.amsu.2015.03.007.

4. Al-Mayoof A, Al-Ani BH. Left-side Amyand hernia: Report of two cases with review of literature. Eur J Pediatr Surg Rep. 20I4;2:63-6. doi: I0.IO55/s-0033-I347I3I.

5. Benavides-De la Rosa DF, López-de-Cenarruzabeitia I, Moreno-Racionero F, Merino-Peñacoba LM, Beltrán de Heredia J. Case report: Amyand's hernia, diagnosis to consider in a routine procedure. Rev Esp Enferm Dig. 2015;I07:708-9.

6. Losanoff JE, Basson MD. Amyand hernia: What lies beneath -A proposed classification scheme to determinate management. Am Surg. 2007;73:1288-90.

7. Losanoff JE, Basson MD. Amyand hernia: A classification to improve management. Hernia. 2008;12:325-6.

8. Cigsar EB, Karadag CA, Dokucu AI. Amyand's hernia: II years of experience. J Pediatr Surg. 2016;5I:I327-9. doi: I0.IoI6/j.jpedsurg.20I5.II.oIO.

9. Sahu D, Swain S, Wani M, Reddy PK. Amyand's hernia: Our experience in the laparoscopic era. J Minim Access Surg 2015;II(2):I5I-3. doi: I0.4103/0972994I.I47368.

Io. Aguirre L, Prieto M, García A, García JM, Sarriugarte A, Colina A. Hernia de Amyand (tipo 2 de Losanoff) diagnosticada preoperatoriamente y tratada mediante hernioplastia con malla biológica. Rev Hispanam Hernia. 20I4;2:I69-I72. Doi.org/IOI6/j.rehah.20I4.04.003. 